\title{
SOJOURN TIMES AND THE EXACT HAUSDORFF MEASURE OF THE SAMPLE PATH FOR PLANAR BROWNIAN MOTION
}

\author{
BY \\ DANIEL RAY(1)
}

This paper contains the proof of a conjecture of Ciesielski and Taylor [1]: Let $X(\tau, \omega)$ be a planar Brownian motion with initial point the origin. Let

$$
T(a, t, \omega)=\int_{0}^{t} V(|X(\tau, \omega)| ; a) d \tau,
$$

where

$$
\begin{aligned}
V(r ; a) & =1, \quad 0 \leqq r<a, \\
& =0, \quad a \leqq r,
\end{aligned}
$$

be the measure of that portion of the time interval $(0, t)$ which the path spends within the circle of radius $a$ about the origin. Let

$$
C(t, \omega)=\{X(\tau, \omega): 0 \leqq \tau \leqq t\}
$$

be the planar set formed by the path for $0 \leqq \tau \leqq t$. Let

$$
\phi(a)=\frac{1}{2} a^{2} \log (1 / a) \log \log \log (1 / a) .
$$

THEOREM 1. With probability one,

$$
\underset{a \rightarrow 0}{\lim \sup } T(a, t, \omega) / \phi(a)=1
$$

for each $t>0$.

THEOREM 2. There is a positive number $\theta$ such that with probability one,

$$
\phi-m(C(t, \omega)) \leqq \theta t
$$

for each $t>0$, where $\phi-m(\cdot)$ is the Hausdorff measure defined by the function $\phi$.

Received by the editors March 5, 1962.

(1) Supported in part by the Alfred P. Sloan Foundation and in part by National Science Foundation grant NSF G-19684. 
Corresponding results for Brownian motion in $k$-space, $k \geqq 3$, were proved by Ciesielski and Taylor in [1], verifying a conjecture of Levy [4].

Theorem 2 follows from Theorem 1 exactly as in [1] by using a density theorem of Rogers and Taylor [5]. Whether or not $\phi-m(C(t, \omega))$ is finite remains an open question however. I am very grateful to S. J. Taylor for pointing out that a supposed proof of this last, originally contained here, was indirect.

The sojourn times

$$
T(E, t, \omega)=\int^{t} V(X(\tau, \omega) ; E) d \tau
$$

where

$$
\begin{aligned}
V(x ; E) & =1, \quad x \in E, \\
& =0, \quad x \in E,
\end{aligned}
$$

of the Brownian path up to time $t$ in the Borel set $E$ define for each $t$ and $\omega$ a measure on Borel sets. Theorem 2 and the fact that Brownian motion has independent increments imply easily that with probability one this measure has a density relative to $\phi$-measure, equal to the constant $\theta$ on the set $C(t, \omega)$ of finite $\phi$-measure, and vanishing outside $C(t, \omega)$. Again the corresponding result holds in higher dimensions. The sojourn time of linear Brownian motion has a density relative to Lebesgue measure $d x$ :

$$
T(E, t, \omega)=\int_{E} \mathscr{T}(x, t, \omega) d x
$$

the "local time" $\mathscr{T}(x, t, \omega)$ being continuous in $(x, t)$ for almost all $\omega$ [6].

Theorem 1 is proved by a technique devised by Knight [3], subdividing the path according to successive passage time; this provides the independence which seems necessary in proving asymptotic results of this type. The method may be applied to other similar situations, as we will indicate after describing the setup more precisely in the next section. In particular, it yields a simple proof of the existence of local time for linear Brownian motion. Such a proof exhibits some interesting properties of local time which will be described elsewhere.

As in [1], Theorem 2 is obtained from Theorem 1 by the use of some density theorems of Rogers and Taylor [5] for Hausdorff measures.

1. The statement and proof of Theorem 1 involve only the radial process $R(\tau, \omega)=|\boldsymbol{X}(\tau, \omega)|$ of our planar Brownian motion, with initial point $R(0, \omega)=0$. Due to the spherical symmetry of Brownian motion, the radial process is Markovian. Denote by $R\left(\tau, \omega_{r}\right)$ the process with the same transition function but initial point $R\left(0, \omega_{r}\right)=r$. 
For the radial process starting at $r$, let

$$
P\left(s, \omega_{r}\right)=\operatorname{Inf}\left\{\tau: \mid R\left(\tau, \omega_{r}\right)=s\right\}
$$

be the first passage time across $s$. A special case [2] of the strong Markov property states that the stopped process $R\left(\tau, \omega_{r}\right), 0 \leqq \tau<P\left(s, \omega_{r}\right)$, and the renewed process $R\left(P\left(s, \omega_{r}\right)+\tau, \omega_{r}\right), \tau \geqq 0$, are independent; and that the renewed process is a copy of $R\left(\tau, \omega_{s}\right), \tau \geqq 0$. This remains true conditional on the event $P\left(s, \omega_{r}\right)$ $<P\left(s^{\prime}, \omega_{r}\right)$, for a third point $s^{\prime}$, since this event depends only on the stopped process. Of course, the conditioning is trivial unless $r$ is between $s$ and $s^{\prime}$, when there is the well known formula

$$
\mathscr{P}\left\{P\left(s, \omega_{r}\right)<P\left(s^{\prime}, \omega_{r}\right)\right\}=\frac{\log \left(s^{\prime} / r\right)}{\log \left(s^{\prime} / s\right)} .
$$

Now choose positive numbers $b, B$, and set $a_{n}=B e^{-b n}, n=0,1, \cdots$. Fix $n$ temporarily. For each path of the radial process, let $t_{v}, v=0,1, \cdots$, be the successive passage times across the points $a_{n-1}, a_{n}, a_{n-1}, \cdots$. To be precise, set

$$
\begin{aligned}
t_{0}(\omega) & =0 \\
t_{2 v+1}(\omega) & =\operatorname{Inf}\left\{\tau: \tau>t_{2 v}(\omega), R(\tau, \omega)>a_{n-1}\right\} \\
t_{2 v}(\omega) & =\operatorname{Inf}\left\{\tau: \tau>t_{2 v-1}(\omega), R(\tau, \omega)<a_{n}\right\}
\end{aligned}
$$

Since the process is recurrent, $t_{v}$ is defined for all positive integers $v$. Define

$$
\begin{aligned}
P_{v}(\omega) & =t_{v+1}(\omega)-t_{v}(\omega), \\
R_{v}(\tau, \omega) & =R\left(t_{v}(\omega)+\tau, \omega\right), \quad 0 \leqq \tau<P_{v}(\omega) .
\end{aligned}
$$

Successive applications of the basic property in the preceding paragraph show that the processes $R_{v}(\tau, \omega)$ are independent copies of a radial process $R\left(\tau, \omega_{r}\right)$ stopped at the passage time across $s$, where if $v=0, r=0, s=a_{n-1}$; if $v$ is positive and even, $r=a_{n}, s=a_{n-1}$; if $v$ is odd, $r=a_{n-1}, s=a_{n}$.

The number of returns from $a_{n-1}$ to $a_{n}$ before crossing $B$ is

$$
N_{n}(\omega)=\operatorname{Max}\left\{v: t_{2 v}(\omega)<P(B, \omega)\right\} \text {. }
$$

$N_{n} \geqq k$ if and only if for $0 \leqq v<k$ each of the independent processes $R_{2 v+1}$ starting at $a_{n-1}$ passes across $a_{n}$ before $B$. Hence using (5),

$$
\mathscr{P}\left\{N_{n} \geqq k\right\}=(1-1 / n)^{k} .
$$

Since the event $N_{n}=k$ is independent of the processes $R_{2 v}$, we have

LEMMA 1. Conditional on the value of $N_{n}$, the processes $R_{2 v}(\tau, \omega)$, $0 \leqq \tau<P_{v}(\omega)$, are independent, $0 \leqq v \leqq N_{n}$; and each is a copy of $R\left(\tau, \omega_{r}\right)$, $0 \leqq \tau<P\left(a_{n-1}, \omega_{r}\right)$, where $r=0$, if $v=0, r=a_{n}$ if $v>0$. 
This decomposition will allow us to describe the pertinent fluctuations of the sojourn times $T\left(a_{n}\right)$ as $a_{n} \rightarrow 0$ in terms of the process $N_{n}$ with parameter $n=1,2, \cdots$. The problem is then much easier to handle, since the latter process turns out to be Markovian.

Indeed, suppose $m>n$, and for $v=0,1, \cdots$, let $N_{n, m, v}$ be the number of returns from $a_{m-1}$ to $a_{m}$ of the process $R_{2 v}$. Since the processes $R_{2 v+1}$ can never reach $a_{m}$,

$$
N_{m}=\sum_{0}^{N_{n}} N_{n, m, \nu}
$$

On the other hand, returns from $a_{k-1}$ to $a_{k}$ for $k \leqq n$ can occur only during intervals $\left[t_{v}, t_{v+1}\right]$ for $v$ odd. Each variable $N_{n, m, v}$ is independent of the behavior of the process during these intervals, and so independent of $N_{k}, k \leqq n$. But then (8) implies that given $N_{n}, N_{m}$ is independent of $N_{k}, k<n$.

The transition function can be computed from the distributions of the independent variables $N_{n, m, v}, m>n$. For $v=0, N_{n, m, 0}$ is just the number of returns from $a_{m-1}$ to $a_{m}$ before reaching $a_{n-1}$ of the process starting at 0 . By the same argument used to derive (7),

$$
\mathscr{P}\left\{N_{n, m, 0} \geqq k\right\}=\left(\frac{m-n}{m-n+1}\right)^{k} .
$$

For $v>0, N_{n m, v} \geqq 1$ if and only if the process $R_{2 v}$ starting at $a_{n}$ reaches $a_{m-1}$ before $a_{n-1} ; N_{n, m, v} \geqq k>1$ if and only if $N_{n, m, v} \geqq 1$ and $k-1$ independent copies of the radial process starting at $a_{m}$ reach $a_{m-1}$ before $a_{n-1}$. Thus for $v>0$,

$$
\mathscr{P}\left\{N_{n, m, v} \geqq k\right\}=\frac{1}{(m-n)}\left(\frac{m-n}{m-n+1}\right)^{k}, \quad k \geqq 1 .
$$

The generating function is given by

$$
\begin{aligned}
E\left\{\sigma^{N_{n, m}, v}\right\} & =(1+(m-n)(1-\sigma))^{-1}, \quad v=0, \\
& =1-(1-\sigma)(1+(m-n)(1-\sigma))^{-1}, \quad v>0 .
\end{aligned}
$$

Finally since the $N_{n, m, v}$ are independent of $N_{n},(8)$ implies

$$
\begin{aligned}
& E\left\{\sigma^{N_{m}} \mid N_{n}\right\} \\
& \quad=(1+(1-\sigma)(m-n))^{-1}\left(1-(1-\sigma)(1+(m-n)(1-\sigma))^{-1}\right)^{N_{n}}
\end{aligned}
$$

LEMMA 2. For the radial process starting at the origin, let $N_{n}$ be the number of returns from $B e^{-(n-1) b}$ to $B e^{-n b}$ before the first passage across $B$. The variables 
$N_{n}, n=1,2, \cdots$, form a Markov chain with the initial value $N_{1}=0$ and with the stationary transition function given by (10).

It is worth noting that, except for the formulas depending on (5), what we have done is independent of the dimension. The main difficulty in proving Theorem 1 for planar Brownian motion is reflected in the fact that the chain $N_{n}$ is null recurrent in this case. The analogous chain in dimension greater than two is ergodic, but for a slightly different choice of the sequence $\left\{a_{n}\right\}$, as in [1], $N_{n}=0$ eventually with probability one. In the linear case, the chain $N_{n}$ is transient, and using only Tchebycheff's inequality one can prove that with probability one the sequence $\left(a_{n-1}-a_{n}\right) N_{n}$ converges to a random variable proportional to the density of the sojourn time at the origin.

2. The decomposition of the radial process given by Lemma 1 breaks up the sojourn time $T\left(a_{n}, P(B, \omega), \omega\right)$ into independent increments: As in [3], set

$$
\begin{aligned}
T_{v}(\omega) & =\int_{t_{2 v}(\omega)}^{t_{2 v+1}(\omega)} V\left(R(\tau, \omega) ; a_{n}\right) d \tau \\
& =\int_{0}^{P_{2 v}(\omega)} V\left(R_{2 v}(\tau, \omega) ; a_{n}\right) d \tau .
\end{aligned}
$$

Then

$$
T\left(a_{n}, P(b, \omega), \omega\right)=\sum_{0}^{N_{n}} T_{v}(\omega),
$$

and by Lemma 1, the summands are independent, conditional on the value of $N_{n}$.

For $v \geqq 1, T_{v}$ is the sojourn time within a circle of radius $a_{n}=B e^{-b n}$ of a Brownian motion starting on the circumference and stopped upon crossing the circle of radius $a_{n-1}=B e^{-b(n-1)}$. Since for Brownian motion, for each $\lambda>0$, $X(t) \rightarrow \lambda^{-1} X\left(\lambda^{2} t\right)$ is a measure preserving transformation, $T_{v}$ has the same distribution as $a_{n}^{2} T$, where

$$
T=T\left(\omega_{1}\right)=\int_{0}^{P\left(e^{b}, \omega_{1}\right)} V\left(R\left(\tau, \omega_{1}\right) ; 1\right) d \tau
$$

is the sojourn time inside the unit circle of a Brownian motion starting on the circumference and stopped upon crossing the circle of radius $e^{b}$.

It is well known that

$$
E\left\{T\left(\omega_{1}\right)\right\}=\int_{|y|<1} G(x, y) d y,|x|=1,
$$

where $G$ is the Green's function for Laplace's equation with boundary values zero on the circle of radius $e^{b}$. Thus $E\left\{T\left(\omega_{1}\right)\right\}=\frac{1}{2} b$. Let $\mu_{k}=E\left\{\left(T\left(\omega_{1}\right)-b / 2\right)^{k}\right\}$. Then

$$
E\left\{T_{v}(\omega)\right\}=\frac{1}{2} b a_{n}^{2}, \quad E\left\{\left(T_{v}(\omega)=\frac{1}{2} b a_{n}^{2}\right)^{k}\right\}=a_{n}^{2 k} \mu_{k} .
$$


Since the $T_{v}$ are independent

$$
\begin{aligned}
E\left\{\left(\sum_{v=1}^{N}\left(T_{v}-\frac{1}{2} b a_{n}^{2}\right)\right)^{4}\right\} & =a_{n}^{8}\left(N \mu_{4}+3 N(N-1) \mu_{2}^{2}\right) \\
& <C N^{2} a_{n}^{8},
\end{aligned}
$$

and by a Tchebycheff type inequality,

$$
\begin{aligned}
\mathscr{P}\left\{\left|\sum_{v=1}^{N} T_{v}-\frac{1}{2} b a_{n}^{2} N\right|>n a_{n}^{2}\right\} \\
<n^{-4} a_{n}^{-8} E\left\{\left(\sum_{v=1}^{N}\left(T_{v}-\frac{1}{2} b a_{n}^{2}\right)\right)^{4}\right\} \\
<C N^{2} n^{-4} .
\end{aligned}
$$

Since the $T_{v}$ are independent conditional on $N_{n}$, (11) implies

$$
\begin{aligned}
\mathscr{P}\left\{\left|T\left(a_{n}, P(B)\right)-T_{0}-\frac{1}{2} b a_{n}^{2} N_{n}\right|>n a_{n}^{2}\right\} \\
\quad=E\left\{\mathscr{P}\left\{\left|\sum_{v=1}^{N_{n}} T_{v}-\frac{1}{2} b a_{n}^{2} N_{n}\right|>n a_{n}^{2} \mid N_{n}\right\}\right\} \\
<C n^{-4} E\left\{N_{n}^{2}\right\} \\
<2 n^{-2} .
\end{aligned}
$$

By the Borel-Cantelli lemma, with probability one as $n \rightarrow \infty$, as in [3],

$$
T\left(a_{n}, P(B)\right)=T_{0}+\frac{1}{2} b a_{n}^{2} N_{n}+O\left(n a_{n}^{2}\right) .
$$

Now $T_{0}(\omega)<P\left(a_{n-1}, \omega\right)$, and by homogeneity, $E\left\{P^{2}\left(a_{n-1}\right)\right\}=C a_{n-1}^{4}<C^{\prime} a_{n}^{4}$. Hence by Tchebycheff's inequality and the Borel-Cantelli lemma,

$$
T_{0}=O\left(n a_{n}^{2}\right)
$$

as $n \rightarrow \infty$ with probability one. This is of course implied by Theorem 4 of [1], but we do not need so strong a result.

3. The standard techniques of Markov chain theory suffice to prove

$$
\underset{n \rightarrow \infty}{\lim \sup }\left(N_{n} / n\right) \log \log n=1
$$

with probability one, as follows.

Using (10) with

$$
\sigma=(1+x)^{-1}<1, x=(\sqrt{N}-\sqrt{\lambda y}) /(m-n+1) \sqrt{\lambda y}
$$

for $\lambda<1$, we get the estimate 


$$
\begin{aligned}
\mathscr{P}\left\{N_{m}\right. & \left.<\lambda y \mid N_{n}=N\right\} \\
& \leqq \sigma^{-\lambda y} E\left\{\sigma^{N_{m}} \mid N_{n}=N\right\} \\
& =(1+x)^{\lambda y} \frac{1+x}{1+x+(m-n) x}\left(1-\frac{x}{1+(m-n+1) x}\right)^{N} \\
& <\exp \left\{\lambda y x-\frac{N x}{1+(m-n+1) x}\right\} \\
& =\exp \left\{-\frac{(\sqrt{n}-\sqrt{\lambda y})^{2}}{m-n+1}\right\} \\
& <\exp \left\{-(1-\sqrt{\lambda})^{2}\right\} \\
& =1-\delta(\lambda)
\end{aligned}
$$

if $N=N_{n} \geqq y \geqq m-n+1>0$.

Under the same restrictions on $y$ and $\lambda$,

$$
\begin{aligned}
\mathscr{P}\left\{N_{m}\right. & >\lambda y\} \\
& \geqq \mathscr{P}\left\{\exists k, n \leqq k<m, N_{k} \geqq y ; N_{m} \geqq \lambda y\right\} \\
& =\sum_{k=n}^{m-1} \mathscr{P}\left\{N_{j}<y, n \leqq j<k ; N_{k} \geqq y ; N_{m} \geqq \lambda y\right\} \\
& \geqq \delta(\lambda) \sum_{k=n}^{m-1} \mathscr{P}\left\{N_{j}<y, n \leqq j<k ; N_{k} \geqq y\right\} \\
& =\delta(\lambda) \mathscr{P}\left\{\exists k, n \leqq k<m, N_{k} \geqq y\right\} .
\end{aligned}
$$

Let $\mu$ be an arbitrary number exceeding one, and choose $\lambda<1, c>1$, such that $\lambda \mu>c$. Using the above and.(5), for $n$ large,

$$
\begin{aligned}
\mathscr{P}\left\{\exists k, c^{n}\right. & \left.\leqq k<c^{n+1}, \quad N_{k} \geqq \mu k \log \log k\right\} \\
& \leqq \mathscr{P}\left\{\exists k, c^{n} \leqq k<c^{n+1}, \quad N_{k} \geqq \mu c^{n} \log \log c^{n}\right\} \\
& \leqq \frac{1}{\delta(\lambda)} \mathscr{P}\left\{N_{c} n+1 \geqq \lambda \mu c^{n} \log \log c^{n}\right\} \\
& =\frac{1}{\delta(\lambda)}\left(1-c^{-(n+1)}\right)^{\lambda \mu c^{n} \log \log c^{n}} \\
& <C n^{-\lambda \mu / c} .
\end{aligned}
$$

By the Borel-Cantelli lemma,

$$
\underset{n \rightarrow \infty}{\lim \sup } N_{n} / n \log \log n \leqq \mu
$$

with probability one. 
On the other hand, suppose $\eta<1$. Choose $c>1$ so that $\eta c<c-1$. For $n=c^{k-1}, m=c^{k}, k=2,3, \cdots$, let $N_{n, m, 0}$ be the number of returns from $a_{m-1}$ to $a_{m}$ before the first passage of $a_{n-1}$, as defined in $\S 1 . N_{n, m, 0}$ depends only on $R(\tau, \omega)$ for $P\left(a_{m-1}, \omega\right)<\tau<P\left(a_{n-1}, \omega\right)$, and as $k$ varies these time intervals are disjoint. Hence by the basic property of $\S 1$, the variables $N_{n, m, 0}$ are independent, $k=2,3, \cdots$.

By (9)

$$
\begin{aligned}
\mathscr{P}\left\{N_{n, m} 0 \geqq \eta\right. & m \log \log m\} \\
& =\left(1+\frac{1}{m-n}\right)^{-\eta m \log \log m} \\
& =\exp \left\{-\eta \frac{m}{m-n} \log \log m\right\} \\
& =\exp \left\{-\eta \frac{c}{c-1} \log (k \log c)\right\} \\
& =C k^{-\eta c /(c-1)} .
\end{aligned}
$$

By the converse of the Borel-Cantelli lemma,

$$
\lim _{k \rightarrow \infty} \sup _{n, m, 0} / m \log \log m \geqq \eta
$$

with probability one. But by (8), $N_{m} \geqq N_{n, m, 0}$ and so

$$
\lim \sup N_{m} / m \log \log m \geqq \eta
$$

with probability one. This together with (15) implies (14).

4. We complete the proof of Theorem 1. By (12), (13), and (14), and since $b n \sim \log \left(1 / a_{n}\right), \log \log n \sim \log \log \log \left(1 / a_{n}\right)$,

$$
\left.\lim _{n \rightarrow \infty} \sup T\left(a_{n}, P B\right)\right) / \phi\left(a_{n}\right)=1
$$

with probability one, for each choice of $B$ and $b$. If $a_{n+1}<a \leqq a_{n}$, then $T(a, P(B)) \leqq T\left(a_{n}, P(B)\right)$, while $\phi(a) \geqq \phi\left(a_{n+1}\right) \sim e^{-2 b} \phi\left(a_{n}\right)$.

Hence

$$
\limsup _{a \rightarrow 0} T(a, P(B)) / \phi(a) \leqq e^{2 b}
$$

with probability one. Since $b$ is arbitrary, $e^{2 b}$ may be replaced by 1 on the right, and since the opposite inequality is trivial,

with probability one.

$$
\lim _{a \rightarrow 0} \sup T(a, P(B)) / \phi(a)=1
$$


Given positive numbers $\varepsilon$ and $\delta$, we can choose $B$ so small that $P(B)<\delta$ with probability exceeding $1-\varepsilon$. Since $t>P(B)$ implies $T(a, t)>T(a, P(B))$,

$$
\lim _{a \rightarrow 0} \sup T(a, t) / \phi(a) \geqq 1
$$

whenever $t>\delta$, with probability at least $1-\varepsilon$. Similarly, for arbitrary positive $A$ we can choose $B$ so large that $P(B)>A$ with probability exceeding $1-\varepsilon$. Thus

$$
\lim _{a \rightarrow 0} \sup T(a, t) / \phi(a) \leqq 1
$$

whenever $t<A$, with probability at least $1-\varepsilon$. And since $\varepsilon, \delta$, and $A$ are arbitrary, Theorem 1 follows.

\section{REFERENCES}

1. Z. Ciesielski and S. J. Taylor, First passage times and sojourn times for Brownian motion in space and the exact Hausdorff measure of the sample path, Trans. Amer. Math. Soc. 103 (1962), 434-450.

2. G. A. Hunt, Some theorems concerning Brownian motion, Trans. Amer. Math. Soc. 81 (1956), 294-319.

3. F. B. Knight, On the random walk and Brownian motion, Trans. Amer. Math. Soc. 103 (1962), 218-228.

4. P. Levy, La mesure de Hausdorff de la courbe du mouvement brownien, Giornale dell Istituto Ital. Attuari 16 (1953), 1-37.

5. C. A. Rogers and S. J. Taylor, Functions continuous and singular with respect to a Hausdorff measure, Mathematika 8 (1961), 1-31.

6. H. Trotter, A property of Brownian motion paths, Illinois J. Math. 2 (1958), 425-433.

MassachusetTs INSTitute OF TeChNOLOGY,

Cambridge, Massachusetts 